\title{
Epidemiology of human immunodeficiency virus in prospective blood donors and testing algorithm assessment with real-time PCR for optimal blood safety practice
}

\begin{abstract}
Background: The challenge of 'safe blood' practice has given rise to several unanswered questions in the practice of immune-hematology and has led to controversies as to which algorithm adopted for HIV testing in prospective blood donors pose the least risk and provide safe blood to recipients of blood transfusion. The aim of this study is to evaluate HIV epidemiology and the outcomes of CDCUMD serial algorithm II for HIV antibody testing in our resource-limited setting by advancing HIV screening beyond serologic procedure.
\end{abstract}

Material and Methods: A total of 140 prospective blood donors were enrolled in the study between November, 2014 and July, 2015 by consecutive sampling technique. Study used HIV antibody screening based on serial algorithm II as the first-line testing protocol followed by ELISA technique. HIV-DNA confirmation and HIV-RNA quantification were carried out on pooled DBS and plasma samples respectively using real-time PCR.

Results: The overall mean age and gender ratio of PBD were 27.9 \pm 7.9years and 1.1:1. Chi square analysis revealed that the PBD had the right knowledge of all the routes of HIV transmission $\left(\chi^{2}\right.$ range: $\left.63.3-212.7, p<0.05\right)$. Study showed an overall HIV prevalence of $2.9 \%$ irrespective of assay technique with inter-category variation in prevalence. Pooled sero-negative DBS samples analysis by real-time PCR showed absence of occult HIV infection among PBD and viral load of confirmed positive blood donors revealed low viraemia (least being 25 copies $/ \mathrm{mL}$ ).

Conclusion: Based on the study findings, HIV rapid antibody testing based on serial algorithm II did not compromise 'safe blood practice'. Adoption of rapid HIV antibody techniques based on serial algorithm II can serve as a surrogate HIV confirmatory technique in PBD where facilities for ELISA or the more costly real-time PCR are unavailable.

Keywords: HIV, epidemiology, blood safety, DBS, testing algorithm, real-time PCR
Volume 4 Issue 6 - 2017

\author{
Fasakin KA, ${ }^{1,2}$ Ifeanyichukwu MO, ${ }^{3}$ Amilo $\mathrm{GI},{ }^{4}$ \\ Agbakoba NR ${ }^{5}$ \\ 'Department of Hematology and Blood Transfusion, Federal \\ Teaching Hospital, Nigeria \\ ${ }^{2}$ Department of Medical Laboratory Science, Afe Babalola \\ University, Nigeria \\ ${ }^{3}$ Department of Immunology, Nnamdi Azikiwe University, \\ Nigeria \\ ${ }^{4}$ Department of Hematology, Nnamdi Azikiwe University, \\ Nigeria \\ ${ }^{5}$ Department of Medical Laboratory Science, Nnamdi Azikiwe \\ University, Nigeria
}

\begin{abstract}
Correspondence: Fasakin Kolawole Asimiyu, Department of Hematology and Blood Transfusion, Federal Teaching Hospital, Ido Ekiti, Ekiti State, Nigeria,

Email fasakin_kolawole@yahoo.co.uk
\end{abstract}

Received: May 24, 2017| Published: July 28, 2017
Abbreviations: Ag-Ab, antigen-antibody; CAP/CTM, COBAS ampliprep/COBAS TaqMan; CAT, category; CDC, centre for disease control and prevention; CLSI, clinical and laboratory standard institute; DBS, dry blood spot; CDNA, complementary deoxyribonucleic acid; DNA, deoxyribonucleic acid; ELISA, enzymelinked immunosorbent assay; FMOH, federal ministry of health; HIP, HIV-1/2 infected partner; HIV, human immunodeficiency virus; HPC: high positive control; IVDA, intravenous drug abuse; K EDTA, tri-potassium ethylene diamine tetra acetic acid; LPC, low positive control; MTCT, mother-to child transmission; MTMS, male-to-male sexual transmission; NBTS, national blood transfusion service; NC, negative control; NEG, negative; NR, non-reactive; OE, occupational exposure; SUNS, sharing of unsterilized needles and syringes; OPBD, overall prospective blood donors; PBD, prospective blood donors; PDBD, paid blood donors; POS, positive; QL, qualitative technique; $\mathrm{QQ}$, quantification technique; QS, quantitative standard; R, reactive; RBD, replacement blood donors; RNA, ribonucleic acid; S \& C , sneezing and coughing; SISO, sharing of infected sharp objects; $\mathrm{SSB}$, sharing of same bed; TIBBP, transfusion of infected blood and blood products; VBD, voluntary blood donors; UMD, university of maryland; WHO, world health organization

\section{Introduction}

Although blood transfusion is one of the known therapeutic interventions that are commonly used in several clinical settings and situations, it is not without associated risks including transmission of human immunodeficiency virus (HIV), viral hepatitis and recent emergent viral threats. ${ }^{1-3}$ Blood safety, over the years, has remained a key issue of major concern in transfusion medicine especially in sub-Saharan Africa. ${ }^{4}$ The transfusion of blood safe from human immunodeficiency virus (HIV) and other blood-borne infections has been the pursuit and priority of international organizations, national health facilities and agencies involved in the practice and regulation of blood transfusion science..$^{1,5-7}$ The fundamental understanding of the far reaching consequences and inestimable cost of unsafe blood, both from the social-economic and healthcare points of view, warrant careful blood donor selection and testing algorithms that guarantee safe blood free from HIV and other blood-borne infections. ${ }^{8}$ The 
World Health Organization (WHO) advocated for optimal blood donor selection that reduces the risk of blood-borne infections including HIV by promoting $100 \%$ voluntary non-remunerated blood donation. ${ }^{9-11}$ Despite this advocacy, majority of blood donors in resource-limited settings are replacement blood donors and paid blood donors which are associated with higher risks of transmitting blood-borne infections including HIV. Several studies have documented the prevalence of $\mathrm{HIV}$ infections in prospective blood donors in various regions of the world. ${ }^{8,12-17}$ Transfusion of blood and blood products has been reported to account for $5-10 \%$ of HIV transmission in sub-Saharan Africa ${ }^{18-19}$ Needless to mention that the paid blood donation system constitutes the category of blood donation with high prevalence of HIV while voluntary blood donation the least. ${ }^{20}$ In many of our clinical settings and international conferences of experts, controversies exist as to which algorithm is best in diagnosing HIV among the existing guidelines while other researchers insist that there is no standardized algorithm for diagnosing HIV. ${ }^{21-25}$ Though some studies have attempted to lay these controversies to rest, ${ }^{26-28}$ more studies are still needed to provide evidence-based findings. Some study reports on high prevalence of HIV infections among prospective blood donors including voluntary blood donors raise pertinent questions on HIV as a threat to blood safety. ${ }^{18}$ What is the best donor recruitment strategy that can help reduce the scourge of post-transfusion HIV to the barest minimum? Can the serial algorithm II in current use in many clinical settings in Nigeria be said to provide safe blood free from HIV infection? Is there possibility of occult HIV infections in those already certified fit for donation on the basis of rapid HIV antibody immunoassay techniques? In resource constraint settings, how reliable is HIV diagnosis made on the basis of serologic procedures (including ELISA) without confirmation through Western blot or molecular assays such as real-time PCR? This study was carried out to provide some evidence-based findings on the effectiveness or noneffectiveness of alternative algorithm for HIV diagnosis in promoting blood safety and reduce inadvertent transmission of HIV through blood transfusion.

Reports by the WHO showed that 108 million blood donations are collected globally every year. About $50 \%$ of these are collected in high-income countries where less than $20 \%$ of the world's population lives. ${ }^{29}$ The average blood donation rate is more than 9times greater in high-income countries than in low income countries. ${ }^{22}$ Current facts showed that only 62 countries get close to $100 \%$ of their national blood supplies from voluntary non-remunerated blood donations with 34 countries still dependent on family replacement blood donors and even paid blood donors for over $75 \%$ of their supply. ${ }^{29}$ Various authors have documented facts on voluntary blood donors (VBD) ${ }^{20,15}$ replacement blood donor (RBD) and paid blood donors (PDBD). ${ }^{30}$ Clinical settings that encourage replacement blood donation at the expense of voluntary blood donation more often than not indirectly encourage paid blood donation. ${ }^{23}$ In most blood transfusion laboratories in lowincome countries, most donors perceive blood donation exercise as a means of meeting life's needs rather than donating to save life. The commercialization of blood donation is in breach of the fundamental principle of altruism which voluntary blood donation enshrines. ${ }^{11}$ With the establishment of more than eighteen National Blood Transfusion Service (NBTS) centers in the six geopolitical zones of Nigeria, it is expected that collaboration of Nigerian Health Institutions with NBTS should boost voluntary blood donations but there are limited published data to exemplify this which informed this study.

CDC-UMD serial algorithm II is an alternative confirmatory guideline for HIV diagnosis in resource constraint settings which involves consecutive performance of HIV by rapid antibody techniques and is thought to compare in effectiveness to more costly algorithms. ${ }^{23}$ It involves the use of the most sensitive rapid immune chromatographic technique (such as Determine) as the first-line testing protocol, the more specific technique (e.g. Unigold) as the next to confirm positive HIV results. In case of sero-discordance, another technique different from the two based on different principle (e.g. Stat-Pak) is used as the tie-breaker. ${ }^{31}$ Some clinical settings prefer the use of parallel HIV testing algorithm prior confirmatory procedures ${ }^{32}$ while others make enzyme-linked immuno-sorbent assay (ELISA) technique the starting point for HIV diagnosis. ${ }^{33}$ There is virtual lack of data on the rapid HIV antibody testing based on serial algorithm II validated with molecular techniques (such as real-time polymerase chain reaction) in HIV diagnosis especially in Ekiti state and that requires evidence-based baseline study.

\section{Materials and methods}

\section{Sample collection and storage}

Up to five milliliters $(5 \mathrm{ml})$ of $\mathrm{K}_{3}$ EDTA anti-coagulated samples were obtained from consented donors. Plasma separated immediately following centrifugation according to Clinical and Laboratory Standard Institute guidelines ${ }^{34}$ (CLSI, 2003) were aliquot into two separate pilot bottles or plain containers for analysis.

\section{Dry blood spot (DBS) sample}

Dry blood spot samples were used for qualitative detection of HIV-DNA as for early infant diagnosis. Ninety-two (92) of the seronegative samples collected into $\mathrm{K}_{3}$ EDTA containers by venipuncture were randomly selected from one hundred and thirty-six (136) prospective blood donors initially screened HIV $1 / 2$ negative and used for HIV 1/2 confirmation by real-time PCR.

\section{DBS pooling technique}

Fifty microliters $(50 \mu 1)$ of each of 20 VBD's samples pooled was placed into plain container. The procedure was repeated for the remaining 54 VBD samples in three batches in pool of 20,20 and 14 . 12 RBD and 6 PDBD samples were pooled separately. After thorough mixing by inversion 8-10times to ensure homogeneity, with the aid of sterile Pasteur pipettes, a drop of blood was placed aseptically to fill the circular, demarcated areas of the dry blood spot (DBS) cards and labeled and allowed to stand for at least 3 hours before being packaged.

\section{Study population}

Prospective blood donors (PBD) recruited by the collaborative efforts of NBTS and Blood Transfusion laboratory of the Federal Teaching Hospital, Ido Ekiti within the study period and some replacement and paid blood donors who volunteered to allow for comparison of outcomes.

\section{Sampling technique}

Consecutive sampling technique was used for this study.

\section{Research subjects exclusion criteria}

Exclusion criteria for the research subjects in this study include pregnancy, non-informed consent and those with evidence of HIV infection.

\section{Questionnaires and informed consent form}

Questionnaires were administered to the prospective blood donors to obtain the demographic characteristics, blood donation history, HIV awareness/risk and other relevant information. Oral and/or 
written consent were obtained from the prospective blood donors before drawing samples from them.

\section{Ethical approval}

Ethical approval for this research study was obtained from the Ethics and Research Committee of the Federal Teaching Hospital, Ido Ekiti.

Serologic diagnosis: The HIV rapid antibody-based serological studies were carried out at the Blood Transfusion laboratory, Federal Teaching Hospital, Ido Ekiti following serial algorithm II guideline. Determine kit (based on immune chromatographic principle), manufactured by Alere Medical Co. Ltd, Chiba, Japan was first used, followed by Unigold kit (from Trinity Biotech, Wicklow, Ireland). Stat-Pak kit (Chembio Diagnostic Systems, NY, USA) was used as tie breaker for sero-discordant sample.

Interpretation based on serial algorithm II:_Concordant results (from both Determine and Unigold) were considered positive while discordant results were first repeated by a senior officer and when confirmed, they were followed up with the third HIV $1 / 2$ test using a tie breaker kit, Stat-pak. Stat-Pak, if positive confirmed a positive result. On the other hand, if it were negative, it confirmed negative results. At the NBTS, HIV Ag-Ab detection was tested for all specimens using commercially available Genscreen Monolisa ${ }^{\mathrm{TM}}$ HIV Ag-Ab ULTRA ELISA kits manufactured by BIORAD ( $3, b d$ Raymond Poincare, 92430 Marnes-la-Coquette-France), according to the manufacturer's instructions. ${ }^{35-37}$

Molecular diagnosis: HIV by COBAS Ampliprep/COBAS TaqMan PCR (Real-time PCR Technique) (Roche Molecular Systems, Pleasanton, CA)

\section{Test procedure for HIV-I RNA quantification}

Specimens and controls were brought to room temperature following initial daily maintenance and priming. Reagent cassettes and disposables were then loaded onto the COBAS AmpliPrep Instrument according to manufacturer's instructions. Sample racks were prepared, then $850 \mu$ of High Positive control (HPC), Low Positive Control (LPC), Negative control (NC) and each specimen was transferred using a micropipette and DNase-free tips into the appropriate sample input tubes (S-tubes). The sample racks with S-tubes and K-tubes were then loaded onto the appropriate rack

Table I Gender ratio and age groups of prospective blood donors position on the COBAS AmpliPrep Instrument.

The COBAS AmpliPrep Instrument was then started using the AMPLILINK software and automated specimen processing to isolate HIV-1 RNA was done. Each set of processed specimens in K-tubes, on K- carriers were then manually transferred to the COBAS TaqMan 48 Analyzer using the K-carrier transporter and the COBAS TaqMan 48 Analyzer run was then started. Automated reverse transcription of the target RNA to generate complementary DNA (cDNA) followed by simultaneous PCR amplification of target cDNA and detection of cleaved dual-labeled oligonucleotide probes specific to the target took place in the COBAS TaqMan 48 Analyzer. The Master Mix reagent contains primers and probes specific for both HIV-1 RNA and HIV-1 QS RNA. The HIV-1 QS was added to each specimen at a known copy number and was carried through the specimen preparation, reverse transcription, PCR amplification and detection steps of cleaved duallabeled oligonucleotide detection probes. The COBAS TaqMan Analyzer or COBAS TaqMan 48 Analyzer calculated the HIV-1 RNA concentration in the test specimens by comparing the HIV-1 signal to the HIV-1 QS signal for each specimen and control. At the end of each run the results were printed out.

\section{Results}

\section{Mean age and gender ratios and age groups of prospective blood donors}

Of the 140 prospective blood donors screened for HIV 1/2 using serial algorithm II guideline, $110(78.6 \%) 23(16.4 \%)$ and $7(5.0 \%)$ were VBD, RBD and PDBD respectively. The overall mean age of PBD screened was 27.9( \pm 7.9$)$. 73(52.1\%) and 67(47.9) of PBD were males and female respectively giving male: female ratio of 1.1:1. According to the PBD categories, 21(91.3\%) and 2(8.7\%) enrolled as RBD, thus giving male: female ratio of 10.5:1 and ranking it the highest. Of the seven PDBD screened, 6(85.7\%) and 1(14.3\%) were males and female, giving male: female ratio of $(6: 1)$. VBD had the least as more females, 63(57.3\%) participated than males, 47(42.7\%) resulting in male: female ratio of $0.75: 1$. Younger donors, 16-25years age group constituted the highest population among the overall $\operatorname{PBD}(49.3 \%)$ and VBD $(51.8 \%)$ respectively, followed by 26-35years, $\operatorname{PBD}(33.6 \%)$ and VBD (30.0\%). The findings among RBD and PDBD were quite different. The 26-35years age group constituted the highest population group among RBD (43.5\%) and PDBD (57.1\%). Table 1 $\&$ Table 2 showed the results.

\begin{tabular}{lllll}
\hline Demographic variables & Overall PBD n(\%) & VBD n(\%) & RBD n(\%) & PDBD n(\%) \\
\hline PBD Screened(n(\%)) & $140(100.0)$ & $110(78.6)$ & $23(16.4)$ & $7(5.0)$ \\
Mean age(Mean \pm SD) & $27.9 \pm 7.9$ & $27.9 \pm 8.4$ & $28.3 \pm 5.8$ & $26.3 \pm 3.1$ \\
Sex & & & & \\
Male & $73(52.1)$ & $47(42.7)$ & $21(91.3)$ & $6(85.7)$ \\
Female & $67(47.9)$ & $63(57.3)$ & $2(8.7)$ & $1(14.3)$ \\
Male: Female Ratio & $1.1: 1$ & $0.75: 1$ & $10.5: 1$ & $06: 01$ \\
Age group & & & & \\
$16-25$ & $69(49.3)$ & $57(51.8)$ & $9(39.1)$ & $3(42.9)$ \\
$26-35$ & $47(33.6)$ & $33(30.0)$ & $10(43.5)$ & $4(57.1)$ \\
$36-45$ & $20(14.3)$ & $16(14.5)$ & $4(17.4)$ & - \\
$46-55$ & $3(2.1)$ & $3(2.7)$ & - & - \\
$56-59$ & $1(0.7)$ & $1(1.0)$ & - & - \\
\hline
\end{tabular}

$\mathrm{n}(\%)$ :Absolute number(Percentage), SD, standard deviation. 
Table 2 Marital status as well as blood donation history of prospective blood donors

\begin{tabular}{lllll}
\hline Demographic variables & Overall PBD n(\%) & VBD n(\%) & RBD n(\%) & PDBD n(\%) \\
\hline Marital status & & & & \\
Single & $82(58.6)$ & $66(60.0)$ & $\mathrm{II}(47.8)$ & $5(7 \mathrm{I} .4)$ \\
Married & $53(37.9)$ & $4 \mathrm{I}(37.3)$ & $10(43.5)$ & $2(28.6)$ \\
Widowed & $4(2.9)$ & $2(I .8)$ & $3(8.7)$ & - \\
Divorced/ Separated & $\mathrm{I}(0.7)$ & $\mathrm{I}(0.9)$ & - & - \\
Blood donation history & & & & \\
First timers & $100(71.4)$ & $89(80.9)$ & $15(65.2)$ & $4(57.1)$ \\
Previous donors & $40(28.6)$ & $2 \mathrm{I}(19.1)$ & $8(34.8)$ & $3(42.9)$
\end{tabular}

$\mathrm{n}(\%)$ :Absolute number(Percentage); SD, standard deviation.

Marital status and blood donation history of prospective blood donors

This study also assessed marital status as well as blood donation history of PBD. Nearly $60 \%(58.6 \%)$ of the PBD were singles while $37.9 \%), 4(2.9), 1(0.7 \%)$ were married, widowed and divorced/ separated respectively. Two-third, 66(60.0\%) were singles. RBD and PDBD also had more singles than married enrolees. 100(71.4\%) of the overall PBD screened were first timers while 40(28.6\%) were previous donors. $89(80.9 \%)$ of VBD were first timers ranking it the highest compared to RBD (65.2\%) and PDBD (57.1\%). Table 3 showed the prospective blood donors' knowledge of the mode of transmission of human immunodeficiency virus before performance of procedure (pre-test). Statistically significant numbers of research volunteers had a perfect knowledge of the mode of transmission of HIV $-1 / 2$ as revealed by the $\chi^{2}$ and $p$ values of all the variables $\left(\chi^{2}\right.$ range: $63.3-212.7, \mathrm{p}<0.05)$ tested.

Table 3 Prospective blood donors' knowledge on mode of transmission of human immunodeficiency virus

\begin{tabular}{|c|c|c|c|c|c|c|}
\hline HIV MoT & Yes N(\%) & No N(\%) & I don't know N(\%) & Total & $\chi^{2}$ & *P value \\
\hline SISO & I32(94.3) & $8(5.7)$ & $0(0.0)$ & $140(100.0)$ & 108.8 & 0 \\
\hline TIBBP & $128(9 \mid .4)$ & $7(5.0)$ & $5(3.6)$ & $140(100.0)$ & 212.7 & 0 \\
\hline SSB & $12(8.6)$ & $|2|(86.4)$ & $7(5.0)$ & $140(100.0)$ & 177.9 & 0 \\
\hline MTMS & $48(34.3)$ & $75(53.6)$ & $17(12.1)$ & $140(100.0)$ & 92.1 & 0 \\
\hline$S \& C$ & $10(7.0)$ & I24(88.6) & $6(4.4)$ & $140(100.0)$ & 192.4 & 0 \\
\hline IVDA & $14(10.0)$ & $100(71.4)$ & $26(18.6)$ & $140(100.0)$ & 93 & 0 \\
\hline HIVIP & $126(90.0)$ & $9(6.4)$ & $5(3.6)$ & $140(100.0)$ & 202.5 & 0 \\
\hline МТCT & $125(89.4)$ & $5(3.6)$ & $10(14.0)$ & $140(100.0)$ & 197.5 & 0 \\
\hline OE & 124(88.6) & $8(5.7)$ & $8(5.7)$ & $140(100.0)$ & 192.2 & 0 \\
\hline SUNS & $127(90.0)$ & $4(2.9)$ & $9(6.4)$ & $140(100.0)$ & 207.7 & 0 \\
\hline STET & $40(28.6)$ & $88(62.9)$ & $12(8.6)$ & $140(100.0)$ & 63.3 & 0 \\
\hline
\end{tabular}

$*_{p}<0.05$ is statistically significant.

\section{Serologic screening, confirmation of HIV serostatus and quantification of prospective blood donors' samples with real-time PCR}

Study showed that $4(2.9 \%)$ of the PBD were seropositive while $136(97.1 \%)$ were sero-negative based on the final result of rapid techniques (using three rapid kits-Determine, Unigold and StatPak) following serial algorithm II guideline. 2(1.8\%) of VBD were seropositive while 108(98.2\%) were sero-negative. Similarly, 1 $(4.3 \%)$ of the RBD was seropositive while $22(95.7 \%)$ were seronegative. Also, 1(14.3\%) of PDBD was seropositive while $6(85.7 \%)$ were sero-negative. Study results confirmed PDBD as high-risk group and VBD as low-risk group of blood donors. Repeat of the procedure with the third generation Biorad Genscreen ULTRA HIV 1/2 Ag-Ab
MONOLISA assay showed similar results. Table 4 showed the results of HIV-1/2 Antibody Testing Based on CDC-UMD Serial Algorithm II. ELISA technique findings and final results of $\mathrm{HIV}-1 / 2$ rapid antibody testing were similar.

Table 5 showed the results of confirmatory and quantification results. Phase 1 of the molecular analyses was mainly confirmation of HIV-1/2 serostatus. The four seropositive samples were confirmed positive with real-time PCR using Roche HIV-1 DNA kit. To validate the specificities of the serologic techniques, DBS samples of 92 $(67.6 \%)$ of the sero-negative blood donors were randomly selected in pools of 20, 20, 20 and 14 (from VBD), 12 (from RBD) and 6 (PDBD) and repeated with COBAS Ampliprep/ COBAS TaqMan (real-time PCR) technique. Results of real-time PCR analysis confirmed sero- 
negativity of all the samples tested. Phase 2 of molecular analyses involved the quantification of the confirmed positive blood donor samples. HIV-1 viraemia was assessed in all the four seropositive donors using quantitative real-time PCR assay. Of the two VBD HIV1 samples assayed for HIV-1 viraemia, the first was 14 copies $/ \mathrm{ml}$ while other showed non-detectable level of HIV-1. The lone samples of the RBD and PDBD were also assessed for HIV-1 viraemia. RBD and PDBD HIV-1 viral load results were 25copies/mL and 461 copies/ $\mathrm{mL}$ respectively. Controls from Roche were run in parallel with the research.

Table 4 Results of HIV-I/2 antibody testing based on CDC-UMD serial algorithm II

\begin{tabular}{|c|c|c|c|c|c|c|c|c|c|}
\hline \multicolumn{10}{|c|}{ HIV-I/2 antibody testing techniques } \\
\hline \multirow{2}{*}{ PBD CAT } & \multirow{2}{*}{$n(\%)$} & \multicolumn{2}{|c|}{ Determine } & \multicolumn{2}{|l|}{ Unigold } & \multicolumn{2}{|c|}{ Stat-Pak } & \multicolumn{2}{|c|}{ Final result } \\
\hline & & $\mathbf{R}$ & NR & $\mathbf{R}$ & NR & $\mathbf{R}$ & NR & POS & NEG \\
\hline OPBD & $140(100.0)$ & $5(3.6 \%) \dagger$ & $135(96.4 \%)$ & $4(2.9 \%)$ & $136(97.1 \%) \dagger$ & - & $\mathrm{I}(0.7 \%)$ & $4(2.9 \%)$ & $136(97.1 \%)$ \\
\hline VBD & $110(100.0)$ & $3(2.7 \%)^{*}$ & $107(97.3 \%)$ & $2(1.8 \%)$ & $108(98.2 \%)^{*}$ & - & $\mathrm{I}(0.9 \%)$ & $2(1.8 \%)$ & $108(98.2 \%)$ \\
\hline RBD & $23(100.0)$ & $\mathrm{I}(4.3 \%)$ & $22(95.7 \%)$ & $\mathrm{I}(4.3 \%)$ & $22(95.7 \%)$ & - & - & $\mathrm{I}(4.3 \%)$ & $22(95.7 \%)$ \\
\hline PDBD & $7(100.0)$ & $\mathrm{I}(\mathrm{I} 4.3 \%)$ & $6(85.7 \%)$ & $\mathrm{I}(\mathrm{I} .3 \%)$ & $6(85.7)$ & - & - & $\mathrm{I}(\mathrm{I} .3 \%)$ & $6(85.7 \%)$ \\
\hline
\end{tabular}

$\dagger=$ Lone discordant sample confirmed sero-negative by tie-breaker, serial algorithm II eliminated this false positivity.

*=Lone discordant VBD sample confirmed sero-negative by tie-breaker, serial algorithm II eliminated this false positivity.

Table 5 Confirmation of HIV serostatus and quantification of positive blood donors' samples with COBAS Ampliprep/COBAS Taqman PCR

\begin{tabular}{|c|c|c|c|c|c|c|c|}
\hline \multirow{4}{*}{$\begin{array}{l}\text { Phase I } \\
\text { Blood Donor Category }\end{array}$} & \multicolumn{7}{|c|}{ HIV-I DNA confirmation following serologic screening } \\
\hline & \multirow{2}{*}{\multicolumn{2}{|c|}{$\begin{array}{l}\text { Serologic screening } \\
\text { Rapid technique }\end{array}$}} & & & \multicolumn{3}{|c|}{ Molecular analysis } \\
\hline & & & \multicolumn{2}{|c|}{ Sandwich ELISA } & & \multicolumn{2}{|l|}{ CAP/CTM(QL)** } \\
\hline & $n(\%)$ & $\begin{array}{l}\text { †HIV-I/2+ } \\
\text { n(\%) }\end{array}$ & $\begin{array}{l}\text { HIV-I/2- } \\
\text { n(\%) }\end{array}$ & $\begin{array}{l}\text { †HIV-I/2+ } \\
\text { n(\%) }\end{array}$ & $\begin{array}{l}\text { HIV-I/2- } \\
\text { n(\%) }\end{array}$ & $\begin{array}{l}\text { †HIV-DNA DT: } \\
\text { n(\%) }\end{array}$ & $\begin{array}{l}\text { HIV-DNA } \\
\text { NDT: } n(\%)\end{array}$ \\
\hline OPBD & $140(100.0)$ & $4(2.9)$ & $136(97.1)$ & $4(2.9)$ & $136(97.1)$ & $4(2.9)$ & $92 *(67.6)$ \\
\hline VBD & $110(100.0)$ & $2(1.8)$ & $108(98.2)$ & $2(1.8)$ & $108(98.2)$ & $2(1.8)$ & $74 *(66.1)$ \\
\hline RBD & $23(100.0)$ & $\mathrm{I}(4.3)$ & $22(95.7)$ & $\mathrm{I}(4.3)$ & $22(95.7)$ & $\mathrm{I}(4.3)$ & $12 *(52.2)$ \\
\hline PDBD & $7(100.0)$ & $\mathrm{I}(14.3)$ & $6(85.7)$ & $\mathrm{I}(14.3)$ & $6(85.7)$ & $\mathrm{I}(\mathrm{I} 4.3)$ & $6(85.7)$ \\
\hline Phase 2 & \multicolumn{7}{|c|}{ HIV-I RNA Quantification by Molecular Assay } \\
\hline HIV Positive Blood Donors & \multicolumn{2}{|l|}{ VBD I } & \multicolumn{2}{|l|}{ VBD 2} & \multicolumn{2}{|l|}{ RBD } & PDBD \\
\hline CAP/CTM(QQ)(Copies/mL) & \multicolumn{2}{|l|}{$14 \mid$} & \multicolumn{2}{|l|}{ NDT } & \multicolumn{2}{|l|}{25} & 461 \\
\hline
\end{tabular}

$\dagger=p<0.05$; DT, detected; NDT, non-detected;VL, viral load, HIV-I/2+: Seropositive HIV-I/2 Antibody; HIV-I/2- = Seronegative HIV-I/2 antibody; HIV-DNA, HIV deoxyribonucleic acid; PCR, polymerase chain Reaction

**=Blood donor samples were confirmed for HIV-I using Roche HIV-I kit.

*ND=HIV-I viraemia was below detectable level or this specific donor had HIV-2 virus in his plasma.

*92, *74,*12 and *6: Randomly selected pooled samples from overall PBD, VBD, RBD and PDBD respectively to authenticate the specificity of serologic procedures.

\section{Statistical evidence of HIV diagnostic performances}

Table 6 showed the statistical representations of the performance of the serologic techniques.

The diagnostic performances of the two serologic techniques were compared using diagnostic odds ratio. All the statistical indices including diagnostic odds ratio showed the accuracy and precision of serial algorithm II testing results and ELISA techniques based on realtime PCR. Sensitivities, specificities, positive predictive values and negative predictive values of both techniques were all $100.0 \%$ based on real-time PCR reference technique. Both false positive and false negatives were zero.

Table 6 Statistical comparison of serologic techniques using COBAS Ampliprep/COBAS Taqman PCR as the gold standard

\begin{tabular}{lllllllllll}
\hline Technique & TP & FP & TN & FN & SS & SP & PPV & NPV & DOR & Prevalence(\%) \\
\hline Rapid HIV-Ab EIA & 4 & 0 & 136 & 0 & 100 & 100 & 100 & 100 & Infinity* & $2.90 \%$ \\
Sandwich ELISA & 4 & 0 & 136 & 0 & 100 & 100 & 100 & 100 & Infinity* & $2.90 \%$ \\
CAP/CTM & 4 & 0 & 136 & 0 & 100 & 100 & 100 & 100 & Infinity* & $2.90 \%$ \\
\hline
\end{tabular}

*Infinity: Perfect test( $F P=0 ; F N=0), T P$, true positives, $S S$, sensitivity, TN, true negatives SP, specificity, FP, false positives, PPV, positive predictive value, FN, false negatives, NNV, negative predictive value, DOR, diagnostic odds ratio, CAP/CTM, COBAS ampliprep/COBAS TaqMan PCR, HIV-Ab. EIA, HIV antibody enzyme immunoassay 


\section{Discussion}

As revealed in Tables $1 \& 2$, dominance of younger age groups (16-25years in VBD), increased number of first timers, and high percentage of volunteers with tertiary education were all due to our collaboration with NBTS which engaged in more active motivational outreaches to institutions and educating them on VBD. The findings also showed that achieving $100 \%$ voluntary blood donation in Nigeria is a great possibility. ${ }^{38} \mathrm{~A}$ study in an Indian blood bank showed achievement of $88.0 \%$ compared to the national average of $39.3 \%$ VBD..$^{39}$ Their success was similarly attributed to active motivational outreach to educational institutes through public lectures, presentations, posters and pamphlets thus recruiting long-term young donors. Several studies have demonstrated recruitment of more male donors than females. ${ }^{40,41}$ Moreover, study showed that careful blood donor selection might contribute to reduction in HIV prevalence. Reports by the World Health Organization also stated that less than $10 \%$ of blood is given by women in resource-poor countries. ${ }^{10}$ The 1:1 gender ratio (male: female ratio) observed in this study among VBD might not be unconnected with more female first timers who became motivated on hearing the benefit of free HIV screening in addition to $\mathrm{HCV}$ and multiple hepatitis B markers screening (results not showed in this study) on volunteering to give blood to safe life. Observance of more singles than married individuals in this study was in contrast with Alfouzan ${ }^{42}$ who reported that married individuals had more knowledge on blood donation. Overall, first timers constituted the larger percentages of all categories of blood donors. The results of the assessment of the PBD' baseline knowledge on the mode of transmission of HIV prior testing (pre-test) showed that prospective blood donors had the right knowledge of all the routes of transmission of HIV ( $\chi^{2}$ range : $63.3-212.7, \mathrm{p}: 0.00$ ). This revealed the outcomes of targeted blood donor's recruitment exercise especially by NBTS, and intensive public enlightenment programmes by the NBTS, State Action Committee on AIDS (SACA), faith-based organizations and tertiary health institutions which gained wide coverage in most parts of Ekiti and neighbouring states.

Overall, both rapid HIV-1/2 antibody testing based on serial algorithm II and the conventional ELISA technique showed HIV-1 prevalence of $2.9 \%$ among PBD although there were inter-category differences in prevalence among PBD. The use of a lone rapid technique in making HIV diagnosis is not recommended except in regions where the prevalence is $>30 \%$ among persons with clinical signs and symptoms of HIV infection and in blood screening and surveillance testing in populations with an HIV prevalence of $>10 \%$ (updated UNAIDS/WHO guideline Strategy I) (WHO, 2004). ${ }^{23}$ A lone false positive (FP) result was obtained from Determine thus resulting in $3.6 \%$ HIV seroprevalence and $80.0 \%$ sensitivity. Should Determine rapid technique been the sole rapid immune chromatographic technique adopted, $20 \%$ (i.e. 1 in 5) of PBD would be misclassified as being reactive for HIV. This had the overall consequence of increasing discard rate of donated units or increasing blood donor rejection rate. However, false positive results based on Determine have been traced to some undisclosed underlying chronic diseases in PBD with possible effects of detecting cross-reacting antibodies as HIV antibodies. By extrapolation, the rejection of PBD or discard of donated units based on seropositivity has an overall impact of enhancing the WHO goal of eliminating all risks associated with blood transfusion. It is worth noting that lower sensitivity for Determine rapid immune chromatographic technique compared to previous study in the same institution ${ }^{31}$ could be traced partly to difference in study design. Previous cohort study on known HIV-positive patients when tested with determines showed
$100.0 \%$ sensitivity in consonance with manufacturer's claim. Current one was a cross-sectional study that focussed on the epidemiology of HIV among prospective blood donors. Hence, determine technique's sensitivity was obtained based on few positive cases confirmed by real-time PCR. Based on serial algorithm, further screening with Unigold confirmed only four seropositive donors (i.e. $2.9 \%$ HIV seroprevalence). Inclusion of Stat-Pak as tie breaker kit resolved the discrepancy as it confirmed $2.9 \%$ HIV seroprevalence. Unlike findings by other researchers which showed that the use of Unigold as tie breaker in a serial algorithm did not resolve the challenge of serodiscordance in HIV diagnosis, ${ }^{21}$ this study showed that substitution of Stat-Pak for Unigold did. Serial algorithm II guideline for rapid HIV antibody testing based on the use of Determine as first-line rapid HIV testing procedure followed by Unigold, and subsequent use of StatPak as tie breaker kit seems to have an increased chance of eliminating false positivity. Similarly, based on overall PBD and category of blood donors, ELISA showed corroborating findings. There were no intercategory variations in HIV prevalence among PBD based on ELISA and real-time PCR assay outcomes. Overall HIV prevalence of $2.9 \%$ among the prospective blood donors was confirmed. This is similar to $2.92 \%$ found by Glynn et al., ${ }^{15}$ and nearly 5 -fold the findings of Mavenyengwa et al. ${ }^{16}$ who reported HIV prevalence of $0.6 \%$ among voluntary blood donors in Namibia ${ }^{16}$ and slightly lower than $3.1 \%$ seroprevalence reported by Buseri et al. ${ }^{8}$ Typically, rapid techniques and ELISA have been the most common techniques adopted in blood donor screening ${ }^{17}$ These results showed the effectiveness of alternative rapid enzyme immunoassay serial algorithm as well as the combined rapid EIA-ELISA algorithm in blood donor screening and HIV diagnosis in resource limited settings. ${ }^{27}$

Furthermore, PDBD had highest HIV prevalence (14.3\%) followed by RBD (4.3\%) and RBD had the least HIV prevalence (1.8\%). There was a statistically significant difference in the prevalence of HIV among the blood donors $(\mathrm{p}<0.05)$. A study by Nwogoh et al..$^{30}$ in South-south Nigeria among PDBD and RBD showed that $7.2 \%$ of PDBD were seropositive for HIV compared to $2.6 \%$ among RBD. ${ }^{30}$ Another study by Buseri et al. ${ }^{8}$ in Osogbo, South-West, showed $1.1 \%$ HIV seroprevalence among $\mathrm{RBD}^{8}$ This study result of $4.3 \%$ HIV prevalence among RBD was over 1.5 -fold higher than HIV seroprevalence reported by Nwogoh et al. ${ }^{30}$ in Benin, South-south, Nigeria and nearly 4 -fold of $1.1 \%$ HIV seroprevalence reported by Buseri et al. ${ }^{8}$ None of the voluntary blood donors screened by them was seropositive. WHO recently evaluated the prevalence of transfusion transmissible infections among VBD and reported HIV prevalence of $1.08 \%$ (inter-quartile range, $0.56-2.69 \%$ ) in low-income countries but $0.12 \%$ (inter-quartile range $0.02-0.34$ ) in middle-income countries. ${ }^{43} \mathrm{HIV}$ prevalence among VBD in this study was nearly three-fold HIV prevalence reported by the WHO. That established reasons for more stringent approach in the effectiveness of the system of educating and selecting VBD. Confirmed sero-negative status of randomly selected pooled samples as revealed by the real-time PCR assay showed absence of occult HIV infection among PBD and raised confidence in the specificity of the testing algorithm recommended by the Centre for Disease Control and Prevention ${ }^{44}$ and the WHO. ${ }^{22,23}$ Other researchers reported failure of WHO recommended two-test diagnostic algorithm ${ }^{24}$ while another group of researchers showed the association of the weak line in tie-breaker test to be associated with false positive rate in the serial algorithm approach. ${ }^{21}$ Moreover, confirmatory assay with real-time PCR showed the blood donors were HIV-1 positive. We could not establish HIV-2 or HIV-1/2 coinfection among the blood donors owing to cost and limited number of seropositive samples. Similar findings of HIV-1 being commonplace 
in Nigeria compared to HIV-2 were observed by other researchers and it was responsible for most global HIV/AIDS epidemics. ${ }^{31} \mathrm{HIV}-1$ viraemia were low in HIV-positive blood donors. The findings of HIV1 viral load of 141 copies $/ \mathrm{mL}$ in VBD, 25 copies/mL and 461 copies/ $\mathrm{mL}$ in RBD and PDBD respectively confirmed the asymptomatic status of the blood donors. Observance of non-detected result from the second VBD analyzed for viral load might imply that the HIV-1 was below detectable level of the real-time PCR. Again, it might mean that this particular VBD was positive for HIV-2 which is less virulent and have lower progression to AIDS compared to HIV-1. ${ }^{12}$ Studies have showed the inverse relationships between HIV viral loads and CD4+ T-lymphocytes..$^{45,46}$ DOR adopted in this study validated the reliability of the results of rapid antibody techniques (based on serial algorithm II). Previous studies recorded similar findings in comparison with ELISA or more advanced techniques. ${ }^{31,47}$

\section{Conclusion}

Stringent donor recruitment strategy should be adopted to reduce HIV endemicity and optimize safety of blood. CDC-UMD serial algorithm II guideline for HIV testing did not compromise the goal of blood safety and substitution of Unigold with Stat-Pak as the tie breaker kit improved the sensitivity of the testing algorithm. This guideline on HIV testing algorithm can be used in resource-limited settings as a surrogate algorithm for the more costly algorithm involving immune-blotting assay or real-time PCR. Inclusion of HIV sero-typing in future studies will enhance HIV-2 diagnosis and give sense of direction to physicians on HIV treatment modalities.

\section{Acknowledgements}

None.

\section{Authors contribution}

FKA designed the study, searched for the literatures and performed the analyses. IMO and Amilo co-designed the study, corrected the manuscript and assessed the statistical analyses. ANR went through the literatures and assessed the statistical tables. All authors read and approved the manuscript.

\section{Conflict of interest}

The Author declares no conflict of interest.

\section{References}

1. Walsh GM, Shih AW, Solh Z, et al. Blood-borne pathogens: a canadian blood services centre for innovation symposium. Transfus Med Rev. 2016;30(2):53-68.

2. Yang TO, Cairns BJ, Reeves GK, et al. Cancer risk among $21^{\text {st }}$ century blood transfusion recipients. Ann Oncol. 2017;28(2):393-399.

3. Pozzetto B, Garraud O. New viral risks in blood transfusion by 2016 . Transfus Clin Biol. 2016;23(1):20-27.

4. Noubiap JJ, Joko WY, Nansseu JR, et al. Sero-epidemiology of human immunodeficiency virus, hepatitis $\mathrm{B}$ and $\mathrm{C}$ viruses, and syphilis infections among first time blood donors in Edea, Cameroon. Int $J$ Infect Dis. 2013;17(10):e832-e837.

5. World Health Organization. Guidelines on assessing donor's suitability for blood donation. Geneva, Switzerland: WHO; 2015.

6. Vun MC, Galang RR, Fujita M, et al. Cluster of HIV infections attributed to unsafe injection practices - Cambodia, December 1, 2014-February 28, 2015. MMWR Morb Mortal Wkly Rep. 2016;65(6):142-145.
7. Chevalier MS, Kuehnert M, Basavaraju SV, et al. Progress Towards Strengthening National Blood Transfusion Services-14 Countries, 20112014. MMWR Morb Mortal Wkly Rep. 2016;65(5):115-119.

8. Buseri FI, Muhibi MA, Jeremiah ZA. Sero-epidemiology of transfusiontransmissible infections among blood donors in Osogbo, South-west, Nigeria. Blood Transfus. 2009;7(4):293-299.

9. World Health Organization. Guidelines on assessing donor's suitability for blood donation. Geneva, Switzerland: WHO; 2015. 118 p.

10. World health organization. Sixty-third world health assembly on viral hepatitis infection. Geneva, Switzerland: WHO; 2001.

11. World health organization. The Melbourne declaration on $100 \%$ voluntary non-remunerated donation of blood and blood components. Geneva, Switzerland: WHO; 2009.

12. Campbell-Yesufu OT, Gandhi RT. Update on human immunodeficiency Virus (HIV)-2 infection. Clin Infect Dis. 2011;52(6):780-787.

13. Japhet MO, Adewumi MO, Adesina OA, et al. High prevalence of HIV p24 antigen among HIV antibody negative prospective blood donors in Ile-Ife, Nigeria. J Immunoassay Immunochem. 2016;37(6):555-563.

14. Ghosh A, Singh V, Chawla SS. Seroprevalence of hepatitis B virus infection among blood donors in a tertiary care hospital Western UP. Sch J App Med Sci. 2015;(3D):1337-1342.

15. Glynn SA, Smith JW, Schreiber GB, et al. Repeat whole-blood and platelet-pheresis donors: Unreported deferrable risks, reactive screening tests, and response to incentive programs. Transfusion. 2001;41(6):736743 .

16. Mavenyengwa RT, Mukesi M, Chipare I, et al. Prevalence of human immunodeficiency virus, syphilis, hepatitis B and $\mathrm{C}$ in blood donations in Namibia. BMC Public Health. 2014;14:424.

17. Guan M. Frequency, Causes, and new challenges of indeterminate results in western blot confirmatory testing for antibodies to human immunodeficiency virus. Clin Vaccine Immunol. 2007;14(6):649-659.

18. Bisetegen FS, Bekele FB, Ageru TA, et al. Transmission-transmissible infections among blood donors at Wolaita Sodo University teaching referral hospital, south Ethiopia. Can J Infect Dis Med Microbiol. 2016;2016:8254343.

19. UNAIDS. Report on the global AIDS Epidemic.Geneva, Switzerland: Joint United Nations Program on HIV/AIDS; 2002.

20. Van der Poel CL, Seifried E, et al. Paying for blood donations: Still a risk? Vox Sang. 2002;83(4):285-293.

21. Shanks L, Siddiqui MR, Kliescikova J, et al. Evaluation of HIV testing algorithms in Ethiopia: the role of the tie-breaker algorithm and weakly reacting test lines in contributing to a high rate of false positive HIV diagnoses. BMC Infect Dis. 2005;15:39.

22. World Health Organization. Service delivery approaches to HIV testing and conselling (HTC): a strategic HTC programme framework. Geneva, Switzerland: WHO; 2012. 69 P.

23. World Health Organization. Rapid HIV tests: guidelines for use in $H I V$ testing and counselling services in resource constrained settings. Geneva, Switzerland: WHO; 2004. 48 P.

24. Klarkowski DB, Wazome JM, Lokuge KM, et al. The evaluation of a rapid In situ HIV confirmation test in a programmes with high failure rate of the WHO HIV two-test diagnostic algorithm. PLoS One. 2009;4(2):e4351.

25. Giraldo R, Fiala C, Perez J, et al. HIV tests and their accuracy. South Africa: Presidential AIDS advisory report; 2000.

26. National Guidelines for HIV and AIDS treatment and care in adolescents and adults. Nigeria: Federal Ministry of Health; 2010. $67 \mathrm{P}$ 
27. Bassey O, Bond K, Adedeji A, et al. Evaluation of the performance of nine HIV rapid test kits (RTKs) for the development of an interim national HIV testing algorithm in Nigeria: Laboratory based Phase I Study. AIDS conference, Washington, USA; 2012.

28. Koblavi-Dème S, Maurice C, Yavo D, et al. Sensitivity and specificity of human immunodeficiency virus rapid serologic assays and testing algorithms in an antenatal clinic in Abidjan, Ivory Coast. $J$ Clin Microbiol. 2001;39(5):1808-1812.

29. World Health Organization. Blood connects us all: Voluntary unpaid blood donations must increase rapidly to meet 2020 goal. Geneva, Switzerland: WHO; 2016.

30. Nwogoh B, Ikpomwen OD, Isoa EM. Donor blood procurement and the risk of transfusion transmissible viral infections in a tertiary health facility in South-South Nigeria. Niger Med J. 2001;52(4):227-229.

31. Fasakin KA, Ajayi OD, Ogunbusuyi BO. Diagnostic Utility of adopting Synergy of Rapid Enzyme Immunoassay-ELISA Alternative Confirmatory Algorithm in a Resource-limited Setting. Research Journal of Life Sciences. 2015;2(1):7-20.

32. Manak MM, Njoku OS, Shutt A, et al. Evaluation of the performance of two HIV-1/2 rapid tests in high and low prevalence populations in Nigeria. J Clin Microbiol. 2015;53(11):3501-3506.

33. Mbachu II, Udigwe G, Joseph I, et al. The evaluation of serial rapid HIV test algorithm in the diagnosis of HIV antibodies among pregnant women in south-east Nigeria. BMC Res Notes. 2015;8:557.

34. Arkin CF, Ernst DJ, Marlar A, et al. Tubes and Additives for Venous Blood Specimen Collection; Approved Standard. 5th ed. 2003;H1A4.16(13):45-46.

35. Weber B, Meier T, Enders G. Fourth generation human immunodeficiency virus (HIV) screening assays with an improved sensitivity for p24 antigen close the second diagnostic window in primary HIV infection. $J$ Clin Virol. 2002;25(3):357-359.

36. Saville RD, Constantine NT, Cleghorn FR, et al. Fourth-generation Enzyme-linked immunosorbent assay for the simultaneous detection of human immunodeficiency virus antigen and antibody. J Clin Microbiol. 2001;39(7):2518-2524.
37. Ly TD, Edlinger C, Vabret A. Contribution of combined detection assays of p24 antigen and anti-human immunodeficiency virus (HIV) antibodies in diagnosis of primary HIV infection by routine testing. $J$ Clin Microbiol. 2000;38(6):2459-2461.

38. World Health Organization. Blood safety and donation: fact sheet No.279. Geneva, Switzerland: WHO; 2008.

39. Sareen R, Gupta GN and Dutt A. Donor awareness: key to successful voluntary donation [version 1 referees: 2 approved reservations]. F1000Research. 2012;1:29

40. Lavanya V, Viswanathan T, Sheeba Malar SA, et al. Prevalence of hepatitis $\mathrm{B}$ virus infection among blood donors with antibodies to hepatitis B core antigen. International Journal of Medicine and Medical Sciences. 2012;4(6):128-137.

41. Olokoba AB, Danburam ASF, Desalu OO, et al. Viral hepatitides in voluntary blood donors in Yola, Nigeria. European Journal of Scientific Research. 2009;31(3):329-324.

42. Alfouzan N. Knowledge, Attitudes, and motivations towards blood donation among king Abdulaziz medical city population. International Journal of Family Medicine. 2014;2014:1-8.

43. World health organization. Blood Safety and Availability Fact Sheet. Geneva, Switzerland: WHO; 2017.

44. Rapid HIV Testing in outreach and other community settingsunited states, 2004-2006. Morbidity and Mortality Weekly Report. 2007;56(47):1233-1237.

45. MacNeil A, Sarr AD, Sankalé JL, et al. Direct evidence of lower viral replication rates in vivo in Human Immunodeficiency Virus Type 2 (HIV2) infection than in HIV-1 infection. J Virol. 2007;81(10):5325-5330.

46. Popper SJ, Sarr AD, Travers KU, et al. Lower human immunodeficiency virus type 2 (HIV-2) reflects the difference in pathogenicity of HIV-1 and HIV-2. J Infect Dis. 1999;180(4):1116-1121.

47. Mbanya D. Use of quality rapid diagnostic testing for safe blood transfusion in resource-limited settings. Clin Microbiol Infec. 2013;19(5):416-421 